\title{
Inhibition of ischemic cardiomyocyte apoptosis through targeted ablation of Bnip3 restrains postinfarction remodeling in mice
}

\author{
Abhinav Diwan, ${ }^{1}$ Maike Krenz, ${ }^{2}$ Faisal M. Syed,, Janaka Wansapura, ${ }^{3}$ Xiaoping Ren, ${ }^{4}$ \\ Andrew G. Koesters, ${ }^{1}$ Hairong Li, ${ }^{1}$ Lorrie A. Kirshenbaum, ${ }^{5}$ Harvey S. Hahn, ${ }^{6}$ Jeffrey Robbins, ${ }^{2}$ \\ W. Keith Jones, ${ }^{4}$ and Gerald W. Dorn II',2 \\ ${ }^{1}$ Center for Molecular Cardiovascular Research and 2 Department of Pediatrics, University of Cincinnati, Cincinnati, Ohio, USA. ${ }^{3}$ Imaging Research Center, \\ Department of Radiology, Cincinnati Children's Hospital Medical Center, Cincinnati, Ohio, USA. ${ }^{4}$ Department of Pharmacology, University of Cincinnati, \\ Cincinnati, Ohio, USA. ${ }^{5}$ Institute of Cardiovascular Sciences, St. Boniface General Hospital Research Centre, Winnipeg, Manitoba, Canada. \\ ${ }^{6}$ Charles F. Kettering Memorial Hospital, Dayton, Ohio, USA.
}

\begin{abstract}
Following myocardial infarction, nonischemic myocyte death results in infarct expansion, myocardial loss, and ventricular dysfunction. Here, we demonstrate that a specific proapoptotic gene, Bnip 3 , minimizes ventricular remodeling in the mouse, despite having no effect on early or late infarct size. We evaluated the effects of ablating Bnip3 on cardiomyocyte death, infarct size, and ventricular remodeling after surgical ischemia/ reperfusion (IR) injury in mice. Immediately following IR, no significant differences were observed between $B n i p 3^{-/-}$and WT mice. However, at 2 days after IR, apoptosis was diminished in Bnip $3^{-/}$periinfarct and remote myocardium, and at 3 weeks after IR, Bnip $3^{-/-}$mice exhibited preserved LV systolic performance, diminished LV dilation, and decreased ventricular sphericalization. These results suggest myocardial salvage by inhibition of apoptosis. Forced cardiac expression of Bnip3 increased cardiomyocyte apoptosis in unstressed mice, causing progressive LV dilation and diminished systolic function. Conditional Bnip3 overexpression prior to coronary ligation increased apoptosis and infarct size. These studies identify postischemic apoptosis by myocardial Bnip3 as a major determinant of ventricular remodeling in the infarcted heart, suggesting that Bnip3 may be an attractive therapeutic target.
\end{abstract}

\section{Introduction}

Of the more than 1 million patients who suffer a myocardial infarction (MI) each year in the United States, one-quarter to onethird will die before receiving medical attention (1) and another one-quarter to one-third of the early survivors will die within the following year (2). Accordingly, current therapeutics are focused on maximizing immediate myocardial salvage by mechanical or pharmacological coronary reperfusion, which is associated with reduced in-hospital mortality (3). Long-term outcome after MI is predicted by LV dysfunction, which is present in approximately $40 \%$ of post-MI patients (4); like the treatment of heart failure (5), chronic management of MI therefore involves neurohormonal blockade (5). However, post-MI care directed toward early reperfusion and late management of ventricular dysfunction does not address the critical intermediate period wherein nonischemic injury can lead to generalized cardiac dilation and progressive systolic dysfunction called ventricular remodeling (6).

Remodeling can be considered from either a mechanical or a molecular perspective (7). In the context of cardiac mechanics, focal myocardial injury after MI locally increases myocardial strain in the border zone during isovolumic systole, resulting in production of reactive oxygen species and stimulating apoptosis of nonischemic cardiomyocytes in an expanding infarct border

Nonstandard abbreviations used: $\mathrm{Bnip} 3, \mathrm{Bcl} 2$ and nineteen-kilodalton interacting protein-3; IR, ischemia/reperfusion; $\alpha$-MHC, $\alpha$-myosin heavy chain; MI, myocardial infarction.

Conflict of interest: The authors have declared that no conflict of interest exists. Citation for this article: J. Clin. Invest. 117:2825-2833 (2007). doi:10.1172/JCI32490. zone (8). A vicious cycle of increasing strain and stress ultimately causes global ventricular dilation, a more spherical geometry, and depressed systolic function, i.e., remodeling (9). Based on this paradigm, infarct patching and cardiac support devices have been employed to prevent remodeling through passive ventricular constraint $(10,11)$. The molecular view is that myocardial injury causes local release of cytokines that stimulate apoptosis $(12,13)$ and degrade myocardial matrix (14) in the border zone. Resulting global ventricular dilation and increasing wall stress in the remote myocardium stimulate hypertrophic pathways that further predispose cardiomyocytes to apoptosis (15-17).

Cardiomyocyte apoptosis is at the intersection of both mechanical and molecular mechanisms for postinfarction remodeling and therefore may be an attractive therapeutic target for breaking the cycle leading to heart failure. The approach of pharmacological caspase inhibition $(18,19)$ can be limited by nonspecific systemic effects, making it advantageous to identify and target specific upstream mediators of ischemia-induced cardiomyocyte death. One such factor is Bnip3 ( $\underline{\mathrm{B}} \mathrm{cl} 2$ and nineteen-kilodalton interacting protein- ; ref. 20 ), a proapoptotic $\mathrm{Bcl} 2$ family member that is transcriptionally upregulated via HIF $\alpha$ during hypoxia in the heart and other tissues (21-24). In tissue culture studies and in vitro perfused hearts, Bnip3 knockdown or dominant inhibition diminishes hypoxic cardiomyocyte death, whereas forced cardiomyocyte expression provokes apoptosis $(23,25,26)$, but its effects in the clinically relevant condition of in vivo cardiac ischemia are unknown. Accordingly, we created mice in which the Bnip3 gene was ablated in the germline or conditionally overexpressed in the heart and studied them at baseline and after myocardial 
A
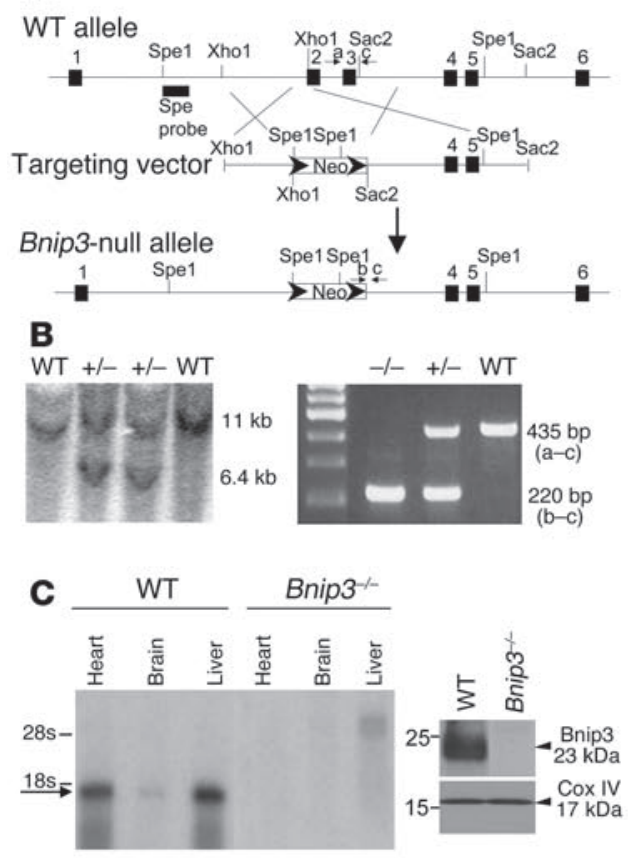

D
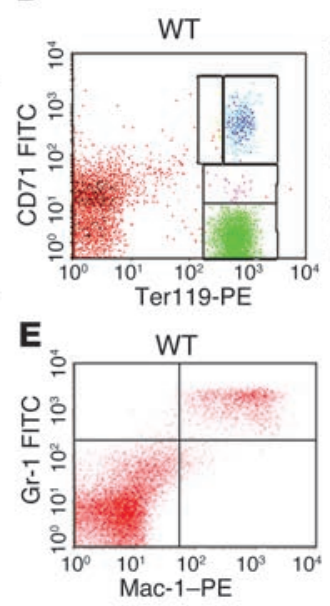

$\mathbf{F}$

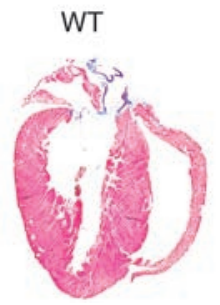

G
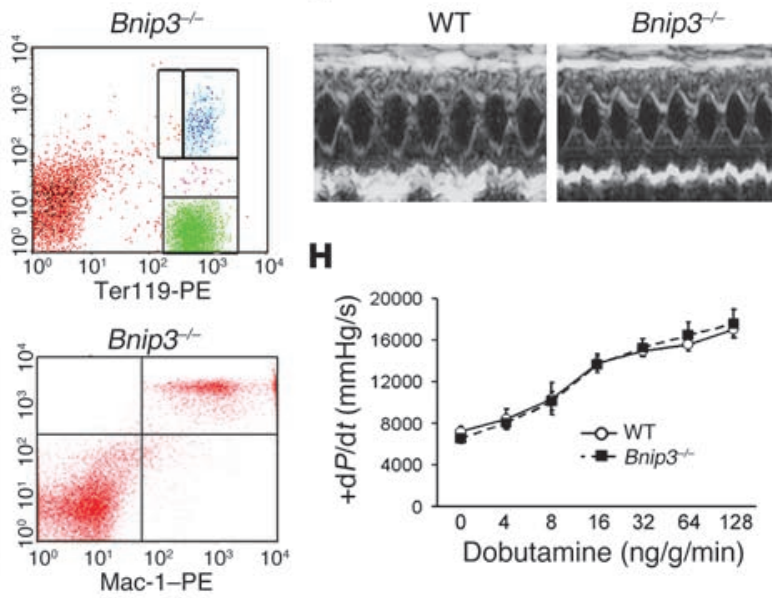

Figure 1

Generation and baseline characteristics of Bnip3-null mice. (A) Schematic of Bnip3 deletion strategy depicting locations of exons 1-6 (black boxes), restriction sites, probe used for Southern blotting (Spe probe), and PCR primers (a-c). (B) Southern blot (left) and PCR (right) screening of Bnip3-targeted mice. (C) Bnip3 multiple tissue Northern blot (left) and immunoblot analysis of mitochondria-enriched myocardial protein (right). (D) Fluorescence cytometry of erythroid Ter119 and CD71 expression in splenocytes. Yellow represents proerythroblasts; blue, basophilic erythroblasts; pink, chromatophilic erythroblasts; green, orthochromatic erythroblasts. (E) Fluorescence cytometry of Gr-1 (granulocyte) and Mac-1 (macrophage) expression in bone marrow cells. (F) Masson trichrome-stained coronal heart sections. (G) M-mode echocardiograms; short-axis view of LV. (H) Invasive hemodynamic analysis of LV contractility at baseline and in response to $\beta$-adrenergic agonist dobutamine $(n=4, \mathrm{WT} ; n=8$, Bnip3---).

ischemia/reperfusion (I/R) injury. Bnip3 ablation reduced postMI myocardial apoptosis by approximately one-half in the infarct border zone and remote myocardium, enhancing cardiac function and minimizing ventricular dilation and sphericalization. Bnip3 overexpression increased myocardial apoptosis with or without ischemia. These findings identify Bnip3 as an important mediator of ischemia-induced myocardial apoptosis and demonstrate therapeutic efficacy for the broad approach of apoptosis inhibition by targeting critical death-effector genes after MI.

\section{Results}

Gene ablation reveals no essential function for Bnip 3 in unstressed mice. A murine model of Bnip 3 ablation was generated using conventional gene targeting through homologous recombination (Figure 1A). The targeting vector containing a neomycin cassette was introduced in place of exons 2 and 3 , thereby truncating the protein prior to the critical BH3 and transmembrane domains (27). Bnip3null mice were identified by Southern blotting (Figure 1B, left) and PCR analysis (Figure 1B, right) of genomic DNA. Bnip3 RNA (Figure 1C, left) and Bnip3 protein (Figure 1C, right) were not detectable in homozygous Bnip3-null mice (Figure 1C), which were born from heterozygous crosses at normal Mendelian ratios (of 77, 13 were $B n i p 3^{+/+}, 43$ were $B n i p 3^{+/}$, and 21 were $B n i p 3^{-/-}$) and showed no increase in mortality or apparent physical abnormalities during fol- low-up for over 14 months. Since gene ablation of other proapoptotic Bcl2 family genes has resulted in striking abnormalities of hematopoietic cells (28-31) that could potentially confound myocardial infarction studies (32-36), we characterized the hematopoietic system in detail. There were no abnormalities in circulating red or white blood cell or platelet counts of Bnip3-null mice (Table 1), and flow cytometric analysis of spleen and bone marrow showed normal proportions of erythroid (Figure 1D), myeloid (Figure 1E), and lymphoid subpopulations (Supplemental Figure 1; supplemental material available online with this article; doi:10.1172/

\section{Table 1}

Peripheral blood counts in Bnip3-null mice

\begin{tabular}{lccc}
\hline & WT $(\boldsymbol{n}=\mathbf{5})$ & Bnip3 $^{--}(\boldsymbol{n}=\mathbf{5})$ & $\boldsymbol{P}$ \\
wbc $\left(\times 10^{3} / \mathrm{ml}\right)$ & $9.7 \pm 0.8$ & $8.6 \pm 0.8$ & 0.368 \\
rbc $\left(\times 10^{6} / \mathrm{ml}\right)$ & $10.2 \pm 0.3$ & $9.8 \pm 0.3$ & 0.372 \\
Hemoglobin $(\mathrm{g} / \mathrm{dl})$ & $15.8 \pm 0.2$ & $15.4 \pm 0.3$ & 0.288 \\
Hematocrit $(\%)$ & $53.5 \pm 1.8$ & $51.6 \pm 0.9$ & 0.359 \\
Platelets $\left(\times 10^{6} / \mathrm{ml}\right)$ & $795 \pm 42$ & $739 \pm 40$ & 0.368 \\
Reticulocytes $(\%)$ & $5.0 \pm 0.3$ & $3.6 \pm 0.7$ & 0.098 \\
\hline
\end{tabular}

Data are presented as mean \pm SEM. $P$ values presented were determined by Student's $t$ test. 


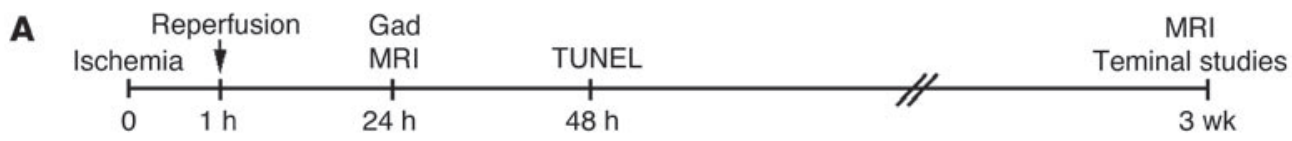

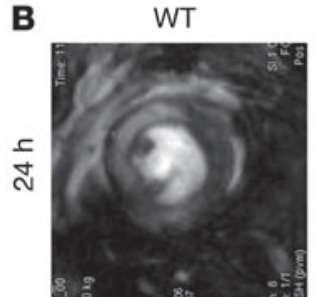

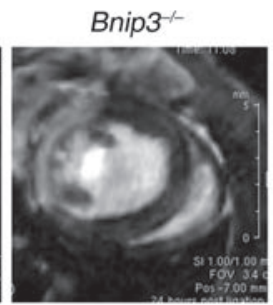

E

WT

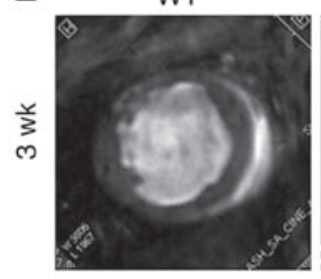

H

WT

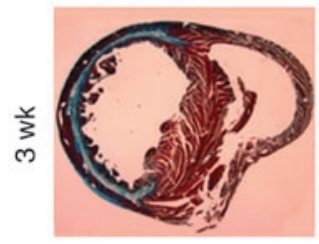

Bnip3 ${ }^{--}$

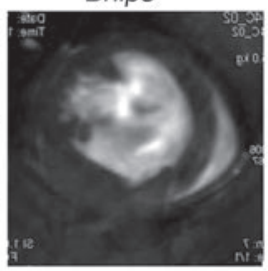

Bnip3 $3^{-1}$

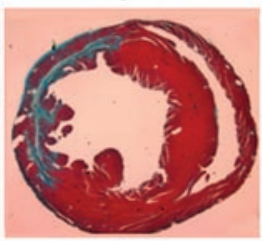

C

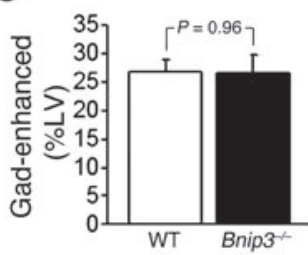

$\mathbf{F}$

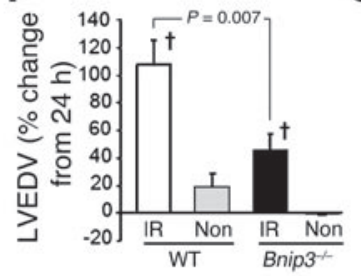

G
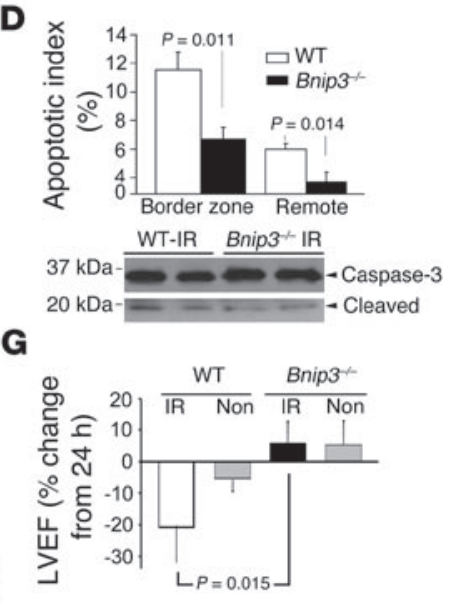

I

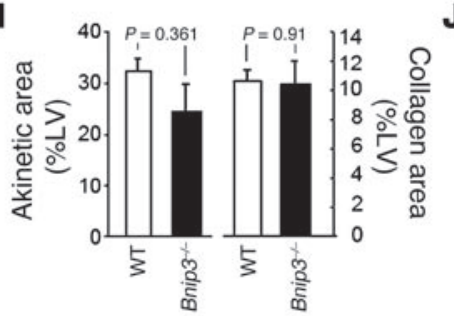

J

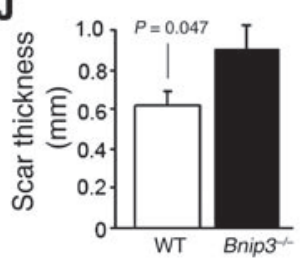

Figure 2

Effects of Bnip3 ablation on in vivo myocardial I/R injury. (A) Schematic depiction of experimental design for in vivo IR studies. Gad, gadolinium. (B) Representative gadolinium-enhanced (white) MRI mid-ventricle end-diastolic images 24 hours after IR. (C) Quantitative infarct size of grouped data $(n=8-9)$. (D) Top: Apoptotic indices (TUNEL) 48 hours after IR $\left(n=6\right.$, WT; $n=7$, Bnip3 $\left.{ }^{-l-}\right)$. Bottom: Representative caspase-3 cleavage studies. (E) Representative mid-ventricle end-diastolic MRI images 3 weeks after IR. Quantitative change in MRI-derived LV end-diastolic volume (LVEDV) (F) and.LV ejection fraction (G) from 24 hours to 3 weeks after IR. ${ }^{\dagger} P<0.05$ vs. nonoperated group (Non). (H) Masson trichrome-stained transverse heart sections (original magnification, $\times 5$ ) 3 weeks after IR. (I) Grouped data for MRI-derived akinetic area (left) and histological collagen scar content (right) of LVs 3 weeks after IR. (J) Scar thickness 3 weeks after IR.

JCI32490DS1). Likewise, Bnip3 ablation did not measurably affect the size, structure, or contractile performance (Table 2, Figure 1, F-H, and Supplemental Table 1) of unstressed hearts. These data demonstrate that germline ablation of Bnip 3 has no detectable baseline effect on the cardiac or hematopoietic systems, indicating that knockout mice are suitable for comparison with WT mice after experimental myocardial I/R.

Decreased periinfarct and remote myocardial apoptosis in Bnip3-null mice. Bnip3 is expressed at very low levels in normal cardiomyocytes but is transcriptionally upregulated in cardiomyocytes subjected to hypoxia/ischemia, wherein it causes apoptosis $(22,23)$. To determine whether Bnip3 ablation would protect in vivo ischemic myocardium from apoptosis, we subjected Bnip3-null mice and their WT syngeneic controls to IR injury by reversible (60-minute) surgical ligation of the left anterior descending coronary artery (Figure 2A). Since the results of sham-operated and nonoperated mice were identical in initial studies ( $n=6$; data not shown), nonoperated mice served as nonischemic controls. MRI with gadolinium enhancement of LV infarcts 24 hours after IR (Figure 2B) showed no differences in acute infarct size (percent of LV) between Bnip3null mice $(29 \% \pm 3 \% ; n=11)$ and WT $(24 \% \pm 3 \% ; n=11 ; P=0.268)$.
When the comparison was restricted to mice having infarct sizes, as revealed by gadolinium enhancement, within our predetermined range for chronic studies of ventricular remodeling (15\%-40\%), there was still no difference (Figure 2C). A comparable extent of initial ischemic myocardial damage in WT and Bnip3-null mice was further indicated by similar, approximately one-third, declines in LV stroke volume and ejection fraction 24 hours after IR (compare values between Tables 2 and 3 ) and similar extent of myocardial necrosis measured by Barbeito-Lopez staining (26\% $\pm 4 \%$ in Bnip3null vs. $24 \% \pm 2 \%$ in WT; $n=4$ pairs; $P=0.722$ ).

To assess the prevalence and distribution of apoptotic myocardial cell death as a function of Bnip3 expression, an additional cohort of 6 pairs of mice underwent IR followed by TUNEL analyses after 48 hours. The rate of apoptosis in the periinfarct region was decreased by approximately one-half in Bnip3-null mice compared with WT (from $11.5 \% \pm 1.3 \%$ to $6.7 \% \pm 0.8 \% ; P=0.011$ ). Although the absolute apoptotic rate was less than that in the periinfarct region, there was a proportionally similar decline in apoptotic index in Bnip3-null myocardium remote from the infarcted area (from $6.0 \% \pm 0.4 \%$ to $3.7 \% \pm 0.7 \% ; P=0.014$ ) (Figure $2 \mathrm{D}$, top). The $19-\mathrm{kDa}$ cleaved fragment of caspase-3, a biochemical marker 


\section{Table 2}

MRI studies of LV geometry and function in Bnip3-null mice

\begin{tabular}{lccc} 
& WT $(\boldsymbol{n}=\mathbf{5})$ & Bnip3 $^{--}(\boldsymbol{n}=\mathbf{6})$ & $\boldsymbol{P}$ \\
LVEDV $(\mu \mathrm{l})$ & $38 \pm 3$ & $42 \pm 5$ & 0.544 \\
LVESV $(\mu l)$ & $9 \pm 1$ & $11 \pm 3$ & 0.144 \\
SV $(\mu l)$ & $28 \pm 2$ & $31 \pm 3$ & 0.526 \\
EF $(\%)$ & $74 \pm 2$ & $77 \pm 4$ & 0.634 \\
SI & $1.98 \pm 0.05$ & $1.75 \pm 0.11$ & 0.128 \\
\hline
\end{tabular}

Data are presented as mean \pm SEM. $P$ values presented were determined by Student's $t$ test. LVEDV, LV end-diastolic volume; LVESV, LV end-systolic volume; SV, stroke volume; EF, ejection fraction; SI, sphericity index.

of apoptosis, was also less prominent in Bnip3-null hearts (Figure $2 \mathrm{D}$, bottom). By comparison, nonischemic mice of either genotype demonstrated low levels of myocardial TUNEL positivity (Bnip3null, $0.36 \% \pm 0.08 \%$ vs. WT, $0.38 \% \pm 0.05 \% ; n=4$ each; $P=0.885$ ). These results reveal protection against apoptosis by Bnip 3 ablation in both the infarct border zone and the remote noninfarcted myocardium after coronary occlusion/reperfusion but no effect on acute infarct size or early post-IR LV ejection performance.

$B n i p 3$ ablation prevents late ventricular remodeling after $I / R$ injury. Infarction of at least $15 \%$ of the LV myocardium is needed to produce ventricular remodeling (diastolic chamber dilation and development of a more spherical geometry) and reduced systolic performance (37). To measure the extent to which Bnip3-mediated postischemic cardiomyocyte apoptosis contributes to ventricular remodeling, and to test whether any myocardial salvage by Bnip3 ablation could favorably impact late ventricular morphometry and function, we reexamined 9 Bnip3-null and 8 WT mice matched for acute infarct sizes between $15 \%$ and $40 \%$ three weeks after IR.

Compared with their respective values 24 hours after IR, WT mice followed for 3 weeks showed a doubling of LV end-diastolic volume (Figure 2, E and F, and Table 3), and an additional 20\% decline in ejection fraction (Figure $2 \mathrm{G}$ and Table 3 ). The LV sphericity index also decreased by approximately $20 \%$, reflecting significantly greater sphericity (Table 3 ). These findings represent the typical structural and functional changes of postinfarction ventricular remodeling (38). In identically treated Bnip3-null mice 3 weeks after IR, chronic infarct size measured either as the extent of scarring (Figure 2, $\mathrm{H}$ and I) or as the region of MRI akinesis (Figure 2I) was the same as that in WT mice. However, LV enddiastolic volume increased by only approximately $40 \%$ in Bnip3-null mice (Figure 2F and Table 3); sphericity did not significantly change (Table 3 ); and ejection fraction remained stable in comparison with their early post-IR values (Figure $2 \mathrm{G}$ and Table 3 ). These results show that Bnip3 ablation attenuates ventricular remodeling and prevents late postinfarction functional deterioration.

Bnip3-null mice exhibited acute and chronic infarct sizes similar to those of WT mice subjected to the same IR protocol. Therefore, the late functional and structural benefits of Bnip3 ablation are not readily attributable to infarct expansion. However, histological scar thickness was significantly greater in Bnip3-null mice (Figure 2J), possibly reflecting a cytoprotective effect at the endocardial and epicardial infarct border zones (Figure $2 \mathrm{H}$ ) that could contribute to preservation of global contractile function.

Because increased wall stress in the hypocontractile infarct border zone has the potential to contribute to progressive ventricular remodeling and contractile dysfunction, even in the absence additional myocardial death/scarring (9), we used MRI myocardial tagging to assess regional wall motion in Bnip3-null and WT mouse hearts 3 weeks after IR. Remote myocardial contraction of Bnip3null mice 3 weeks after IR, assessed using MRI tagging as midwall circumferential strain $\left(E_{\mathrm{cc}}\right)$, was significantly better than that of WT mice $(-12.2 \pm 1.0$ in Bnip3-null, $n=8$, vs. $-8.9 \pm 0.6$ in WT, $n=7 ; P=0.005)$, whereas the periinfarct region showed only a nonsignificant trend toward better function (-6.3 \pm 0.5 in Bnip3null vs. $-4.9 \pm 0.8$ in WT; $P=0.146)$. These results provide further support for a benefit of Bnip3 ablation in noninfarcted myocardium of IR hearts and are consistent with current and previous observations of increased cardiomyocyte apoptosis in both the infarct border zone and noninfarcted ventricular wall of human and experimental MI $(13,39,40)$.

Forced cardiac expression of Bnip 3 induces apoptotic ventricular remodeling. While ischemic induction of Bnip3 gene expression in the heart and other tissues is widely accepted (21-23, 41-43), in vitro evidence suggesting that increased Bnip3 protein expression alone is sufficient to cause cardiomyocyte apoptosis (23) is not easily reconciled with results suggesting that an additional death signal is required for Bnip3 activation $(25,26)$. We considered that forced cardiomyocyte Bnip3 expression could help address this issue: If basal cardiomyocyte apoptosis was observed in unstressed in vivo mouse hearts overexpressing Bnip3, then increased expression alone can be sufficient to cause cardiomyocyte death, as was previously observed with the closely related $\mathrm{BH} 3$-only factor Nix/ Bnip3L $(44,45)$. Accordingly, we created and analyzed $\alpha$-myosin heavy chain-driven ( $\alpha$-MHC-driven) Bnip3-transgenic mice. Expression of Bnip3 beginning in the neonatal period $(44,46)$ resulted in an approximately 50 -fold increase in immunoreactive Bnip3 in 8-week-old hearts, with no change in the relative abundance of major cardiac antiapoptotic $\mathrm{Bcl} 2$ family proteins, $\mathrm{Bcl} 2$ and Bcl-xl (Figure 3A). Mice overexpressing Bnip3 as neonates were viable but developed progressive $\mathrm{LV}$ dilation and correspondingly diminished systolic performance as assessed by echocardiography at 10 and 40 weeks of age (Figure 3, B-E) or by invasive hemody-

\section{Table 3}

MRI studies of LV geometry and function after I/R in Bnip3-null mice

\begin{tabular}{|c|c|c|c|c|c|c|c|}
\hline & \multicolumn{3}{|c|}{ WT IR $(n=8)$} & \multicolumn{3}{|c|}{ Bnip3-/- IR ( $(n=9)$} & \multirow[t]{2}{*}{$P$} \\
\hline & $24 \mathrm{~h}$ & $21 \mathrm{~d}$ & $\% \Delta$ & $24 \mathrm{~h}$ & $21 \mathrm{~d}$ & $\% \Delta$ & \\
\hline LVEDV $(\mu \mathrm{l})$ & $36 \pm 3$ & $72 \pm 6^{A}$ & $108 \pm 17$ & $46 \pm 3$ & $67 \pm 7^{A}$ & $46 \pm 11$ & 0.007 \\
\hline LVESV $(\mu \mathrm{l})$ & $16 \pm 1^{B}$ & $43 \pm 5^{A}$ & $181 \pm 41$ & $26 \pm 3^{B}$ & $37 \pm 7$ & $35 \pm 20$ & 0.005 \\
\hline SV $(\mu \mathrm{l})$ & $19 \pm 2^{B}$ & $30 \pm 2^{A}$ & $66 \pm 19$ & $19 \pm 1^{B}$ & $30 \pm 1^{A}$ & $56 \pm 7$ & 0.645 \\
\hline $\mathrm{EF}(\%)$ & $54 \pm 3^{B}$ & $42 \pm 3^{A}$ & $-20 \pm 7$ & $44 \pm 4^{\mathrm{B}}$ & $49 \pm 6$ & $11 \pm 8$ & 0.015 \\
\hline SI & $1.94 \pm 0.03$ & $1.53 \pm 0.02^{\mathrm{A}}$ & $-21 \pm 1$ & $1.75 \pm 0.06$ & $1.65 \pm 0.03$ & $-5 \pm 3$ & $<0.001$ \\
\hline
\end{tabular}

Data are presented as mean \pm SEM. $P$ values listed are for the comparison of percent change $(\% \Delta)$ from 24 hours to 21 days for Bnip3 ${ }^{-1-}$ IR versus WT IR by Student's $t$ test. AP $<0.05$ for comparison of 21 days to 24 hours within each group by paired Student's $t$ test. ${ }^{B} P<0.05$ vs. respective nonoperated group (see Table 1) by Student's $t$ test. 
A

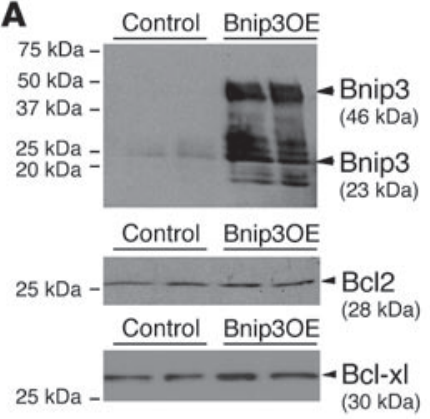

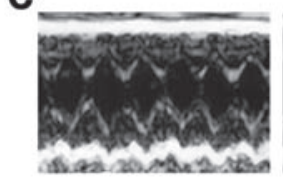

B Control

(10 wk)
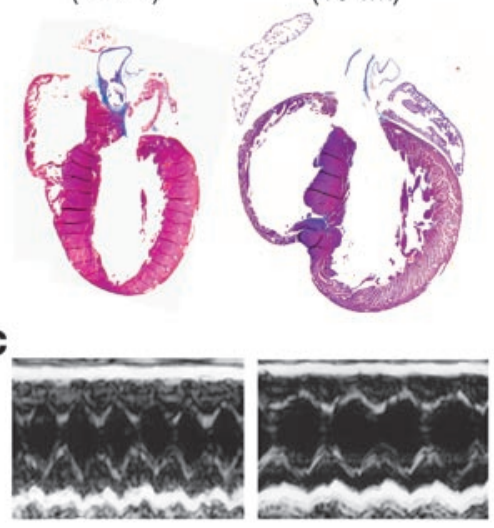
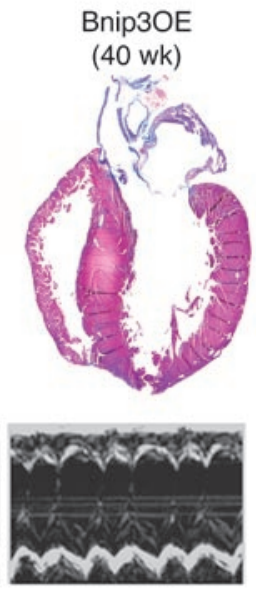

D

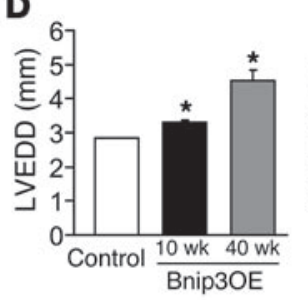

E

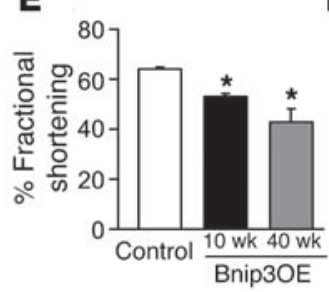

$\mathbf{F}$

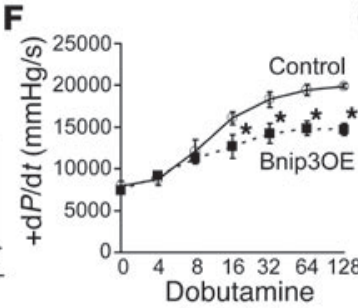

G

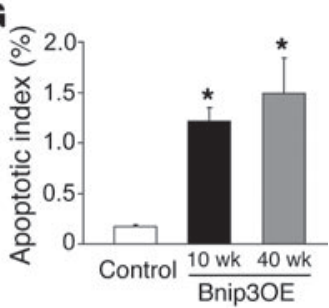

\section{Figure 3}

Studies of cardiac Bnip3 overexpression from the neonatal period. (A) Representative Western blots of myocardial Bnip3, Bcl2, and Bcl-xl in mice with neonatal Bnip3 overexpression (Bnip3OE) and controls. (B) Masson trichromestained coronal heart sections. Original magnification, $\times 2$. (C) Short-axis M-mode echocardiograms. (D and E) Echocardiographically determined LV end-diastolic diameter (LVEDD) and fractional shortening as a function of age $(n=7$ controls, $n=5$ Bnip3 OE at 10 and 40 weeks). (F) Invasively determined LV contractility at baseline and with $\beta$-adrenergic stimulation at 10 weeks ( $n=3$ / group). (G) TUNEL-determined apoptotic indices ( $n=4 /$ group). ${ }^{*} P<0.05$ vs. controls. namic measures at 10 weeks (Figure 3F). Cardiac enlargement was confirmed in terminal studies of 40-week-old mice by gravimetric measures (heart weight/body weight: $8.7 \pm 0.9 \mathrm{mg} / \mathrm{g}$ in Bnip3 overexpressors vs. $5.7 \pm 0.3 \mathrm{mg} / \mathrm{g}$ in controls; $P=0.017)$. Bnip3-overexpressing hearts exhibited rates of apoptosis (i.e., TUNEL staining) 5 - to 10 -fold greater than controls, measured at either 10 or 40 weeks of age (Figure $3 \mathrm{G}$ ). These results indicate that chronically elevated cardiomyocyte Bnip3 protein is sufficient, without added ischemic stress, to cause indolent cardiomyocyte apoptosis in normal hearts, producing LV dilation and contractile dysfunction.

We previously observed synergy between normal postnatal cardiac growth and cardiomyocyte apoptosis induced by the Bnip3related BH3-only factor Nix/Bnip3L (44). To explore whether normal cardiac growth might similarly be acting as a death signal for Bnip3 in neonatal overexpressors, we used doxycycline to suppress tet- $\alpha$ MHC-Bnip 3 transgene activity until the mice were fully grown at 8 weeks of age and then withdrew it to induce adult cardiac Bnip3 expression (Figure 4, A and B) $(44,46)$. As was the case with adult mice overexpressing Nix, ventricular dilation in adult Bnip3 overexpressors was not as striking as that in neonates but was sufficient to result in significant chamber enlargement and depressed ejection performance (Figure 4C). Thus, at high expression levels, there appears to be no absolute requirement for posttranslational Bnip3 activation to produce cardiomyocyte apoptosis in the in vivo heart. Nevertheless, we observed that ischemia can contribute to Bnip3-mediated apoptosis, as cardiomyocyte apoptosis and caspase-3 cleavage were measurably increased in adult Bnip3 overexpressors 48 hours after coronary artery ligation, likely contributing to greater infarct size in these mice (Figure 4, D-F).

\section{Discussion}

The current studies show that postischemic LV remodeling can be attenuated by targeting a critical proapoptotic factor that is spe- cifically expressed in response to ischemia. The general approach of trying to prevent heart failure by inhibiting apoptosis was given mechanistic credence by Wencker et al. (47), who, by expressing a conditionally active caspase in the heart, demonstrated that low levels of cardiomyocyte apoptosis are sufficient to cause ventricular dilation and contractile dysfunction. However, the optimal targets for antiapoptotic interventions in various cardiac syndromes have been elusive, and nonspecific approaches have inherent limitations (48). Herein, we examined whether the hypoxia-regulated BH3-only protein Bnip3 (21-24) might provide such a target. Genetic ablation of Bnip3 decreased postischemic myocardial apoptosis by approximately $50 \%$, suggesting that Bnip3, with or without additional proapoptotic factors, plays a substantial and pathophysiologically meaningful role in the apoptosis that occurs in non- and periinfarct regions of ischemic hearts. These results provide direct evidence that cardiomyocyte apoptosis is an important causal mechanism for postinfarction ventricular remodeling and therefore represents a worthwhile therapeutic target.

The general cellular mechanisms by which Bnip3 induces apoptosis are well established: Via a carboxyterminal membrane-spanning domain, Bnip3 localizes to mitochondria and increases outer mitochondrial membrane permeability (likely through an interaction with Bax or Bak; refs. 49, 50), initiating the intrinsic caspase cascade via cytoplasmic release of cytochrome $c(27,51,52)$. However, there is some divergence in the literature regarding the precise events that lead to Bnip3-mediated apoptosis in hypoxic cardiomyocytes. Although Bnip3 transcription is unequivocally increased by hypoxia $(22,23)$ via the HIF- $1 \alpha$ transcription factor pathway (24), there are different views regarding the necessity for posttranslational activation. Some studies indicate that Bnip3 is a cytoplasmic or loosely associated mitochondrial protein and that hypoxia and acidosis are required, in addition to transcriptional upregulation, to activate Bnip3 and produce cardiomyo- 


\section{A Attenuated $\alpha-\mathrm{MHC}$ promoter

a-MHC promoter
$\longmapsto \#$ TetO

B
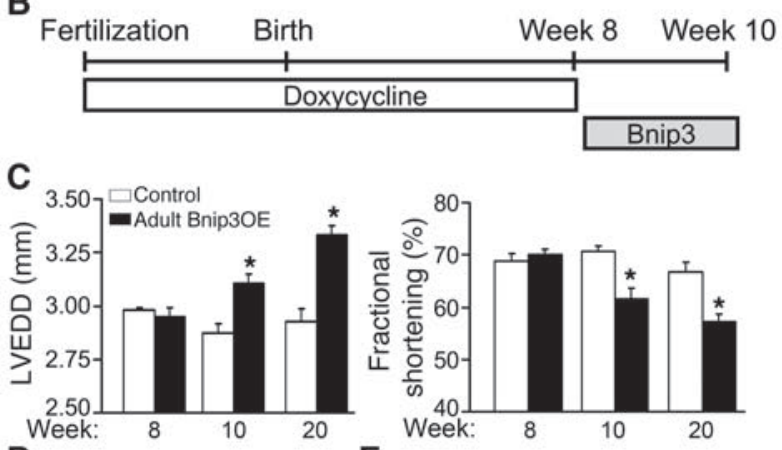

D

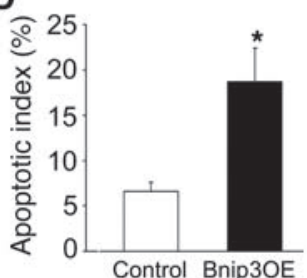

E
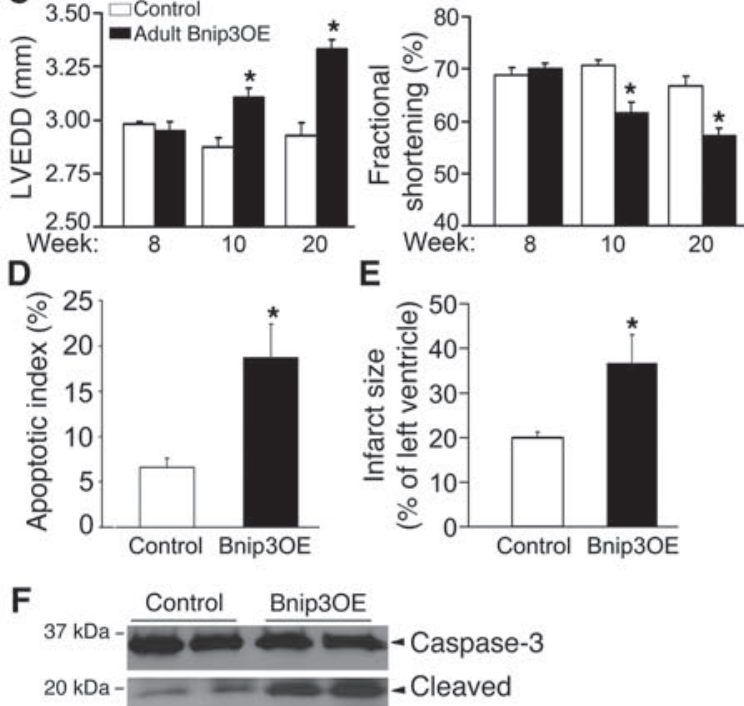

G

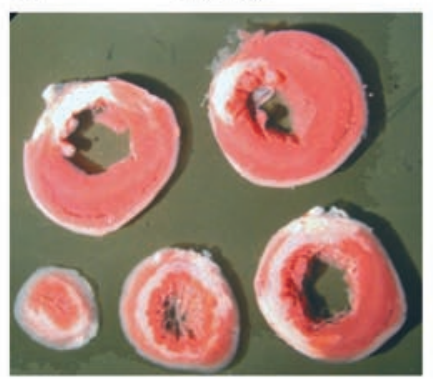

Adult Bnip3OE

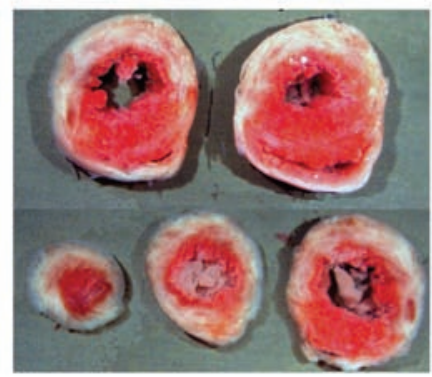

cyte apoptosis $(26,53)$. Others have observed that Bnip3 is tightly associated with mitochondria and that increased expression is sufficient, in the absence of superimposed hypoxia/acidosis, to cause cardiomyocyte apoptosis $(23,25)$. We have observed that both naturally occurring and forcibly overexpressed Bnip3 is found exclusively in a subcellular particulate fraction enriched in mitochondria in cultured cell models and in in vivo mouse hearts (data not shown). Furthermore, we found that overexpression of Bnip3 in otherwise normal (i.e., nonischemic) mouse hearts produced low levels of cardiomyocyte apoptosis that, over time, led to ventricular dilation and systolic impairment. Thus, our findings support the idea that a requirement for hypoxia/acidosis to activate Bnip3 and cause apoptosis is not absolute and that transcriptional increases alone can have significant pathophysiological effects. It should also be noted that Bnip3 has been implicated in nonapoptotic cell death, via opening of the mitochondrial per-

\section{Figure 4}

Studies of conditional Bnip3 overexpression in adult mice. (A) Double conditional transgenesis strategy (see text for details). (B) Schematic of experimental design. (C) Echocardiographically determined LV end-diastolic diameter (left) and LV fractional shortening (right) before Bnip3 induction (week 8) and 2 weeks (week 10) and 12 weeks after induction (week 20); $n=10$ pairs at 8 and 10 weeks and $n=5$ pairs at 20 weeks. (D) TUNEL-derived apoptotic indices in infarcted mice. (E) Quantitative infarct size after 48 hours ( $n=5-6 /$ group). (F).Caspase-3 cleavage studies. (G) Triphenyltetrazolium chloride-stained cardiac cross-sections 48 hours after coronary occlusion. Original magnification, $\times 1 .{ }^{*} P<0.05$ vs. controls.

meability transition pore $(54,55)$, and although the percent LV necrosis did not differ in postischemic Bnip3-null and WT mice in our studies, the current results do not rule out possible effects of Bnip3 on necrotic cell death.

The temporal association between cardiac/cardiomyocyte ischemia and apoptosis has long suggested a pathological role for apoptosis in myocardial ischemic or reperfusion injury $(56,57)$. $\mathrm{I} / \mathrm{R}$ activates both the extrinsic death receptor and intrinsic mitochondrial pathways, and there is evidence that both pathways can contribute to myocardial injury. For the extrinsic pathway, death ligands are released from ischemic cardiomyocytes or inflammatory cells (58) and induce apoptosis via death receptor activation. Indeed, a loss-of-function mutation of the Fas death receptor in mice decreases postischemic apoptosis and infarct size (59). Likewise, a preliminary report suggested that the gene knockout of Bid, a proapoptotic $\mathrm{Bcl} 2$ family factor bridging the death receptor and mitochondrial apoptosis pathways (60), diminishes infarct size and enhances function (61). We do not believe that the current findings relate to inhibition of extrinsic pathway apoptosis, as there is no known crosstalk between Bnip3 and death receptor signaling, and we were careful to exclude an effect of Bnip3 ablation on circulating white cells and on their splenic and bone marrow progenitors, which could be a source of proapoptotic cytokines. Thus, it is likely that Bnip3 modulates only apoptosis transduced via the intrinsic pathway in myocardial ischemia/infarction (62).

Genetic ablation of Bnip3 diminished late ventricular remodeling but did not affect early or late infarct size in our studies. This result contrasts with cardiac overexpression of antiapoptotic $\mathrm{Bcl} 2$, which is reported to reduce infarct size by one-half to two-thirds $(63,64)$. There are important differences between these 2 studies that may explain the different results: Transgenic overexpression of antiapoptotic $\mathrm{Bcl} 2$ shifts the balance strongly toward cell survival prior to the ischemic insult and can therefore affect infarction size. Since Bnip3 is expressed at extremely low levels in nonischemic myocardium (Figure 3A, control lane), we speculate that knocking out a gene that is not significantly expressed prior to induction by ischemia has no effect on infarct size. However, Bnip3 is powerfully induced in hypoxic cardiomyocytes $(21-23,26)$, and its ablation would therefore be cardioprotective for those myocytes that survive the ischemic insult long enough to transcriptionally upregulate and express it, as in the infarct border zone. This protective effect diminished according to the distance from the infarction, likely because the hypoxic stimulus for Bnip3 expression decreases along with the stimulus for cardiomyocyte apoptosis. We suggest that myocardial salvage may be enhanced by reducing both infarct size and late remodeling, perhaps through combined inhibition of extrinsic and intrinsic apoptotic pathways. 
As discussed above, the mechanisms of LV remodeling involve a feed-forward cycle of cardiomyocyte apoptotic and necrotic death, cardiomyocyte slippage and changes in the myocardial interstitium, chamber dilation and decreased wall thickness, and increases in local and global wall stress leading to systemic and local release of pathological neurohormones and cytokines. The current findings show that, without affecting acute or chronic infarct size, reduction of apoptosis by approximately $50 \%$ in the infarct penumbra and remote myocardium substantially attenuates $L V$ remodeling ( $L V$ diastolic dimension and sphericalization), dramatically enhances systolic function (LV ejection fraction and end-systolic dimension), and improves contraction in the noninfarcted wall (midwall circumferential strain). Although the prognostic significance of preserved ejection fraction is sometimes disputed in human heart failure (65), end-systolic volume may be a particularly relevant endpoint, as it reflects both cardiac geometry and contractile function. Indeed, in human studies of remodeling reversal by resynchronization therapy, a reduction in LV end-systolic volume of $10 \%$ was considered significant because it predicted lower mortality and morbidity in heart failure (66). In our studies, absolute LV end-systolic volume in Bnip3-null mice was reduced by $16 \%$ three weeks after IR compared with that in WT controls, consistent with a meaningful improvement in this, as well as the other, parameters. Bnip3 ablation also preserved wall thickness in the scarred segments, which is independently associated with improved function in experimental studies (67).

In summary, these studies identify an important role for Bnip3 as a mediator of post-I/R myocardial apoptosis and late ventricular remodeling and demonstrate that ablation of Bnip3 minimizes ventricular remodeling, despite having no effect on early or late infarct size. An approach of myocardial preservation through early reperfusion and minimization of cardiomyocyte death thereafter can be posited to have certain advantages over attempts to regenerate dead or dying myocardium. Salvaged myocardium is available immediately, during the acute infarct stage when hemodynamics are the least stable, and current myocardial regeneration strategies are limited by inadequate functional contribution of regenerated cardiomyocytes (68). Bnip3 and other proapoptotic factors that may be specifically induced by myocardial ischemia are attractive as therapeutic targets, in combination with revascularization therapy and neurohormonal antagonists, to limit postinfarction myocardial decompensation and prevent development of ischemic cardiomyopathy.

\section{Methods}

Generation and characterization of Bnip3-null mice. Mouse studies were approved by the University of Cincinnati Institutional Animal Care and Use Committee. Bnip3-null mice were generated by replacing exons 2 and 3 (NCBI reference sequence NM_009760) with a neomycin resistance cassette. Recombinants were identified via Spe1 digestion and Southern blotting using a $5^{\prime}$ probe external to the targeting vector. PCR was employed for screening using 3 primers (5'-TGTGGCTGAGAGTCAGTGGTC-3'; 5'-TTGCAAGTCTAGGAGTCAGTT-3'; 5'-GTGGATGTGGAATGTGTGCG-3') to generate a 435-bp WT allele product and a 220-bp Bnip3-null allele product under the following conditions: $94^{\circ} \mathrm{C}$ for 30 seconds, $60^{\circ} \mathrm{C}$ for 30 seconds, $72^{\circ} \mathrm{C}$ for 90 seconds; 33 cycles. Bnip3-null mice were compared with age- and sex-matched WT controls from the common parental strain. Complete blood counts were determined with potassium EDTA anticoagulant. Flow cytometric analysis for CD71 transferrin receptor, Ter119 glycophorin A, CD3, CD19, CD4, and CD8 was performed on freshly isolated spleen and bone marrow cells as described previously (31). Two-dimensional guided M-mode echocardiography was performed in unsedated mice as described previously (69). Invasive hemodynamic studies were performed on anesthetized spontaneously breathing 8- to 12-weekold mice using 1.4 French Millar solid-state catheters (70).

In vivo $I / R$ modeling. Mice were anesthetized with sodium pentobarbital (90 mg/kg, i.p.), intubated with PE-90 tubing (Warner Instruments), and ventilated using a mouse mini-ventilator (Harvard Apparatus) with room air supplemented with oxygen. The respiratory rate was set between 100 and 105 per minute, and $\mathrm{PO}_{2}, \mathrm{PCO}_{2}$, blood $\mathrm{pH}$, and body temperature were maintained within normal limits throughout the procedure as previously described (71). ECG electrodes were placed subcutaneously and data recorded using a PowerLab (ADInstruments). A lateral thoracotomy ( $1.5 \mathrm{~cm}$ left lateral incision between the $2 \mathrm{nd}$ and $3 \mathrm{rd}$ ribs) was then performed to provide exposure of the left anterior descending coronary artery (LAD) while avoiding rib and sternum resection, retraction, and rotation of the heart. The 2 major vascular bundles in the vicinity were coagulated using a microcoagulator (Medical Industries). An 8-0 nylon suture was placed around the LAD 2-3 $\mathrm{mm}$ from the tip of the left auricle and a piece of soft silicon tubing ( $0.64 \mathrm{~mm}$ inside diameter, $1.19 \mathrm{~mm}$ outside diameter) placed over the artery. Coronary occlusion was achieved by tightening and tying the suture (72). Two of the 20 Bnip3-null mice that underwent reversible coronary occlusion surgery died, compared with 12 of 29 WT mice $(P=0.111$ by Fisher exact test $)$.

After 1 hour of coronary occlusion, the suture was untied and left in place. Ischemia was confirmed by visual observation (i.e., pallor) and by continuous ECG monitoring (ST segment changes) and reperfusion by reversal of these effects. Mice without ECG changes indicative of successful and timely I/R were excluded from these studies. The chest was closed in layers using 7-0 polypropylene sutures and the mice allowed to regain consciousness in a warm chamber with $100 \%$ oxygen supplied.

MRI. MRI was performed on a Bruker 7.0 Tesla, $300 \mathrm{~mm}$ bore system located at the Imaging Research Center, Cincinnati Children's Hospital. A gradient insert (internal diameter, $120 \mathrm{~mm}$ ) capable of generating a maximum of $388 \mathrm{mT} / \mathrm{m}$ was used. Mice were anesthetized with continuous inhaled isoflurane ( $2 \%$ by volume) administered via a custom-made nose cone. Constant body temperature of $37^{\circ} \mathrm{C}$ was maintained using a thermocouple/heater system. A custom-made, single-turn solenoid radio frequency coil was used for data acquisition. Imaging was performed with prospective gating for ECG (SA Instruments Inc.) monitored using surface electrodes connected to front paws. A series of gradient echo scout images was generated to determine the long axis of the heart. Cine shortaxis images were then planned from the long-axis scouts. Cine images of the short axis were obtained using a gradient echo (FLASH) sequence. The following imaging parameters were used: TE/TR, 2.6/8.7 ms; number of k-space lines per R-R, 1 ; slice thickness, $1 \mathrm{~mm}, \mathrm{NEX}$, 4; flip, 30 ; FOV, $34 \times 34 \mathrm{~mm}^{2}$; matrix, $256 \times 256$; resolution, $0.13 \times 0.13 \times 1 \mathrm{~mm}^{3}$. Five to 6 contiguous slices were obtained. For contrast-enhanced imaging, a 0.3- to $0.6-\mathrm{mmol} / \mathrm{kg}$ bolus of gadolinium DTPA (Gd-DTPA) was injected i.p. before the mouse was placed in the magnet. High T1 weighting was obtained using a flip angle of $40^{\circ}$ for contrast-enhanced images. Gadolinium enhancement is reported to accurately measure infarct size on MRI (73-76). To validate this, $11 \mathrm{WT}$ mice underwent IR injury and comparative gadolinium-enhanced MRI and triphenyltetrazolium chloride (TTC) staining, which showed a strong correlation (Supplemental Figure 2). Cine images were used to calculate the ejection fraction, ventricular volumes, and the fractional area of contrast enhancement and wall thinning. Image processing and data analysis were performed using software developed at the Imaging Research Center using the IDL programming platform (IDL 6.2; ITT Visual Information Solutions). 
Myocardial tagged magnetic resonance assessment. Tagged images were generated using 5 sinc-modulated $100 \mathrm{~ms}$ square RF pulses. Horizontal and vertical tags were applied in separate acquisitions and were combined in post-processing. Tag separation was $0.8 \mathrm{~ms}$. Myocardial strain analysis was performed using the harmonic phase of the tagged images (Diagnosoft Plus; Diagnosoft Inc.).

Myocardial scar assessment. Hearts subjected to I/R injury were perfusion fixed in $10 \%$ neutral buffered formalin at 3 weeks after I/R injury and subjected to trichrome and picrosirius red staining. Myocardial scar area was quantitated on picrosirius red-stained sections using Scion Image (Scion Corp.). Evaluation of myocardial necrosis was performed using the Barbeito-Lopez trichrome stain as described previously (77).

Generation and characterization of Bnip3-overexpressing mice. A tetracycline-responsive binary $\alpha$-MHC transgene system allowing suppression of transgene expression with doxycycline (78) was used for temporally defined cardiomyocyte-specific expression of the human Bnip3 cDNA. $\alpha M H C m i n T e t O B n i p 3$ mice were bred with $\alpha$ MHCtTA mice, and the double heterozygotes (tTA/MHCminTetOBnip3) were studied with either neonatal or adult expression, as described previously (46). Control mice consisted either of genetically identical littermates with continued doxycycline suppression or TetOBnip3 mice. There were no differences between the 2 control groups.

Bnip3 protein studies. Myocardial extracts were prepared by homogenization of ventricular tissue in a buffer containing $10 \mathrm{mM}$ HEPES pH 7.2, 320

1. Zheng, Z.J., Croft, J.B., Giles, W.H., and Mensah, G.A. 2001. Sudden cardiac death in the United States, 1989 to 1998 . Circulation. 104:2158-2163.

2. Thom, T., et al. 2006. Heart disease and stroke statistics - 2006 update: a report from the American Heart Association Statistics Committee and Stroke Statistics Subcommittee. Circulation. 113:e85-e151.

3. Gibson, C.M. 2004. NRMI and current treatment patterns for ST-elevation myocardial infarction. Am. Heart J. 148:S29-S33.

4. Kober, L., et al. 1995. A clinical trial of the angiotensin-converting-enzyme inhibitor trandolapril in patients with left ventricular dysfunction after myocardial infarction. Trandolapril Cardiac Evaluation (TRACE) Study Group. N. Engl. J. Med. 333:1670-1676.

5. Antman, E.M., et al. 2004. ACC/AHA guidelines for the management of patients with ST-elevation myocardial infarction - executive summary: a report of the American College of Cardiology/ American Heart Association Task Force on Practice Guidelines (Writing Committee to Revise the 1999 Guidelines for the Management of Patients With Acute Myocardial Infarction). Circulation. 110:588-636

6. Pfeffer, M.A., and Braunwald, E. 1990. Ventricular remodeling after myocardial infarction. Experimental observations and clinical implications. Circulation. 81:1161-1172.

7. Ratcliffe, M.B. 2002. Non-ischemic infarct extension: A new type of infarct enlargement and a potential therapeutic target. J. Am. Coll. Cardiol. 40:1168-1171.

8. Pimentel, D.R., et al. 2001. Reactive oxygen species mediate amplitude-dependent hypertrophic and apoptotic responses to mechanical stretch in cardiac myocytes. Circ. Res. 89:453-460.

9. Jackson, B.M., et al. 2002. Extension of borderzone myocardium in postinfarction dilated cardiomyopathy. J. Am. Coll. Cardiol. 40:1160-1167.

10. Kelley, S.T., et al. 1999. Restraining infarct expansion preserves left ventricular geometry and function after acute anteroapical infarction. Circulation. 99:135-142.

11. Blom, A.S., et al. 2005. Cardiac support device modifies left ventricular geometry and myocardial structure after myocardial infarction. Circulation.
mM sucrose, $3 \mathrm{mM} \mathrm{MgCl}_{2}, 25 \mathrm{mM} \mathrm{Na}_{2} \mathrm{P}_{4} \mathrm{O}_{7}, 1 \mathrm{mM}$ DTT, $5 \mathrm{mM}$ EGTA, $20 \mathrm{mM} \mathrm{NaF}$, and $2 \mathrm{mM} \mathrm{Na}_{3} \mathrm{VO}_{4}$ with protease inhibitor cocktail tablet (Roche). Protein extracts were subjected to SDS-PAGE, and anti-Bnip3 (Abcam), anti-Bcl2, anti-Bcl-xl, and anti-cleaved caspase- 3 antibodies (Cell Signaling Technology) were used for immunodetection.

Statistics. Results are expressed as mean \pm SEM. Statistical differences were assessed with the unpaired 2-tailed Student's $t$ test for 2 experimental groups, paired Student's $t$ tests for serial evaluation, and 1-way ANOVA for multiple groups, using SigmaStat (SPSS) software. A nonparametric test was applied when the data were not normally distributed. A 2-tailed $P$ value of less than 0.05 was considered statistically significant.

\section{Acknowledgments}

This work was supported by National Heart, Lung, and Blood Institute grants HL59888, HL77101, HL80008, HL87871, and HL69779.

Received for publication April 24, 2007, and accepted in revised form July 25, 2007.

Address correspondence to: Gerald W. Dorn II, Molecular Cardiovascular Research, 231 Albert Sabin Way, ML 0839, Cincinnati, Ohio 45267-0839, USA. Phone: (513) 558-3065; Fax: (513) 5583438; E-mail: dorngw@ucmail.uc.edu.
112:1274-1283.

12. Olivetti, G., et al. 1997. Apoptosis in the failing human heart. N. Engl. J. Med. 336:1131-1141.

13. Saraste, A., et al. 1997. Apoptosis in human acute myocardial infarction. Circulation. 95:320-323.

14. Wilson, E.M., et al. 2003. Region- and type-specific induction of matrix metalloproteinases in postmyocardial infarction remodeling. Circulation. 107:2857-2863.

15. Sadoshima, J., and Izumo, S. 1993. Mechanical stretch rapidly activates multiple signal transduction pathways in cardiac myocytes: potential involvement of an autocrine/paracrine mechanism. EMBO J. 12:1681-1692.

16. Dorn, G.W. 2005. Physiologic growth and pathologic genes in cardiac development and cardiomyopathy. Trends Cardiovasc. Med. 15:185-189.

17. Dorn, G.W., and Force, T. 2005. Protein kinase cascades in the regulation of cardiac hypertrophy. J. Clin. Invest. 115:527-537. doi:10.1172/ JCI200524178.

18. Yaoita, H., Ogawa, K., Maehara, K., and Maruyama, Y. 1998. Attenuation of ischemia/reperfusion injury in rats by a caspase inhibitor. Circulation. 97:276-281.

19. Chandrashekhar, Y., Sen, S., Anway, R., Shuros, A., and Anand, I. 2004. Long-term caspase inhibition ameliorates apoptosis, reduces myocardial troponin-I cleavage, protects left ventricular function, and attenuates remodeling in rats with myocardial infarction. J. Am. Coll. Cardiol. 43:295-301.

20. Boyd, J.M., et al. 1994. Adenovirus E1B 19 kDa and Bcl-2 proteins interact with a common set of cellular proteins. Cell. 79:341-351.

21. Bruick, R.K. 2000. Expression of the gene encoding the proapoptotic Nip3 protein is induced by hypoxia. Proc. Natl. Acad. Sci. U. S. A. 97:9082-9087.

22. Galvez, A.S., et al. 2006. Distinct pathways regulate proapoptotic Nix and BNip3 in cardiac stress. J. Biol. Chem. 281:1442-1448.

23. Regula, K.M., Ens, K., and Kirshenbaum, L.A. 2002. Inducible expression of BNIP3 provokes mitochondrial defects and hypoxia-mediated cell death of ventricular myocytes. Circ. Res. 91:226-231.

24. Sowter, H.M., Ratcliffe, P.J., Watson, P., Greenberg, A.H., and Harris, A.L. 2001. HIF-1-dependent regulation of hypoxic induction of the cell death fac- tors BNIP3 and NIX in human tumors. Cancer Res. 61:6669-6673.

25. Hamacher-Brady, A., et al. 2007. Response to myocardial ischemia/reperfusion injury involves Bnip3 and autophagy. Cell Death Differ. 14:146-157.

26. Kubasiak, L.A., Hernandez, O.M., Bishopric, N.H., and Webster, K.A. 2002. Hypoxia and acidosis activate cardiac myocyte death through the Bcl-2 family protein BNIP3. Proc. Natl. Acad. Sci. U. S. A. 99:12825-12830.

27. Yasuda, M., Theodorakis, P., Subramanian, T., and Chinnadurai, G. 1998. Adenovirus E1B-19K/BCL-2 interacting protein $\mathrm{BNIP} 3$ contains a $\mathrm{BH} 3$ domain and a mitochondrial targeting sequence. J. Biol. Chem. 273:12415-12421.

28. Bouillet, P., et al. 1999. Proapoptotic Bcl-2 relative Bim required for certain apoptotic responses, leukocyte homeostasis, and to preclude autoimmunity. Science. 286:1735-1738.

29. Bouillet, P., et al. 2002. BH3-only Bcl-2 family member Bim is required for apoptosis of autoreactive thymocytes. Nature. 415:922-926.

30. Ranger, A.M., et al. 2003. Bad-deficient mice develop diffuse large B cell lymphoma. Proc. Natl. Acad. Sci. U. S. A. 100:9324-9329.

31. Diwan, A., et al. 2007. Unrestrained erythroblast development in $\mathrm{Nix}^{-/-}$mice reveals a mechanism for apoptotic modulation of erythropoiesis. Proc. Natl. Acad. Sci. U. S. A. 104:6794-6799.

32. Bajaj, A.K., et al. 1989. Limitation of myocardial reperfusion injury by intravenous perfluorochemicals. Role of neutrophil activation. Circulation. 79:645-656.

33. Entman, M.L., et al. 1991. Inflammation in the course of early myocardial ischemia. FASEB J. 5:2529-2537.

34. Iversen, P.O., and Sorensen, D.R. 2007. A murine model for studying hematopoiesis and immunity in heart failure. Methods Mol. Biol. 360:269-275.

35. van Amerongen, M.J., Harmsen, M.C., van Rooijen, N., Petersen, A.H., and van Luyn, M.J. 2007. Macrophage depletion impairs wound healing and increases left ventricular remodeling after myocardial injury in mice. Am. J. Pathol. 170:818-829.

36. van Beusekom, H.M., et al. 1993. Histology after stenting of human saphenous vein bypass grafts: observations from surgically excised grafts 3 to 320 
days after stent implantation. J. Am. Coll. Cardiol. 21:45-54.

37. Fieno, D.S., et al. 2004. Infarct resorption, compensatory hypertrophy, and differing patterns of ventricular remodeling following myocardial infarctions of varying size. J. Am. Coll. Cardiol. 43:2124-2131.

38. Cheng, A., et al. 2006. Passive ventricular constraint prevents transmural shear strain progression in left ventricle remodeling. Circulation. 114:I79-I86.

39. Sam, F., et al. 2000. Progressive left ventricular remodeling and apoptosis late after myocardial infarction in mouse heart. Am. J. Physiol. Heart Circ. Physiol 279:H422-H428.

40. Palojoki, E., et al. 2001. Cardiomyocyte apoptosis and ventricular remodeling after myocardial infarction in rats. Am. J. Physiol. Heart Circ. Physiol. 280:H2726-H2731.

41. Guo, K., et al. 2001. Hypoxia induces the expression of the pro-apoptotic gene BNIP3. Cell Death Differ. 8:367-376.

42. Zhang, S., et al. 2007. Evidence of oxidative stressinduced BNIP3 expression in amyloid beta neurotoxicity. Brain Res. 1138:221-230.

43. Leo, C., Horn, L.C., and Hockel, M. 2006. Hypoxia and expression of the proapoptotic regulator BNIP3 in cervical cancer. Int. J. Gynecol.Cancer. 16:1314-1320.

44. Syed, F.M., et al. 2005. Proapoptotic effects of caspase-1/interleukin-converting enzyme dominate in myocardial ischemia. Circ. Res. 96:1103-1109.

45. Yussman, M.G., et al. 2002. Mitochondrial death protein Nix is induced in cardiac hypertrophy and triggers apoptotic cardiomyopathy. Nat. Med. 8:725-730

46. Galvez, A.S., et al. 2007. Cardiomyocyte degeneration with calpain deficiency reveals a critical role in protein homeostasis. Circ. Res. 100:1071-1078.

47. Wencker, D., et al. 2003. A mechanistic role for cardiac myocyte apoptosis in heart failure. J. Clin. Invest. 111:1497-1504. doi:10.1172/JCI200317664.

48. Chandrashekhar, Y. 2005. Role of apoptosis in ventricular remodeling. Curr. Heart Fail. Rep. 2:18-22.

49. Bouillet, P., and Strasser, A. 2002. BH3-only proteins - evolutionarily conserved proapoptotic Bcl-2 family members essential for initiating programmed cell death. J. Cell Sci. 115:1567-1574.

50. Kubli, D.A., Ycaza, J.E., and Gustafsson, A.B. 2007. Bnip3 mediates mitochondrial dysfunction and cell death through Bax and Bak. Biochem. J. 405:407-415.

51. Chen, G., et al. 1997. The E1B 19K/Bcl-2-binding protein Nip3 is a dimeric mitochondrial protein that activates apoptosis. J. Exp. Med. 186:1975-1983.

52. Chen, G., et al. 1999. Nix and Nip3 form a subfamily of pro-apoptotic mitochondrial proteins. J. Biol. Chem. 274:7-10.

53. Graham, R.M., et al. 2004. A unique pathway of cardiac myocyte death caused by hypoxia-acidosis. J. Exp. Biol. 207:3189-3200.

54. Vande, V.C., et al. 2000. BNIP3 and genetic control of necrosis-like cell death through the mitochondrial permeability transition pore. Mol. Cell. Biol. 20:5454-5468.

55. Webster, K.A., Graham, R.M., and Bishopric, N.H. 2005. BNip3 and signal-specific programmed death in the heart. J. Mol. Cell. Cardiol. 38:35-45.

56. Fliss, H., and Gattinger, D. 1996. Apoptosis in ischemic and reperfused rat myocardium. Circ. Res. 79:949-956.

57. Gottlieb, R.A., Burleson, K.O., Kloner, R.A., Babior, B.M., and Engler, R.L. 1994. Reperfusion injury induces apoptosis in rabbit cardiomyocytes. J. Clin. Invest. 94:1621-1628.

58. Jeremias, I., et al. 2000. Involvement of CD95/ Apo1/Fas in cell death after myocardial ischemia. Circulation. 102:915-920.

59. Lee, P., et al. 2003. Fas pathway is a critical mediator of cardiac myocyte death and MI during ischemia-reperfusion in vivo. Am. J. Physiol. Heart Circ. Physiol. 284:H456-H463.

60. Yin, X.M. 2000. Signal transduction mediated by $\mathrm{Bid}$, a pro-death Bcl-2 family proteins, connects the death receptor and mitochondria apoptosis pathways. Cell Res. 10:161-167.

61. Foo, R.S.-Y, Mani, K., and Kitsis, R.N. 2005. Death begets failure in the heart. J. Clin. Invest. 115:565-571. doi:10.1172/JCI200524569.

62. Baetz, D., et al. 2005. Nuclear factor-kappaB-mediated cell survival involves transcriptional silencing of the mitochondrial death gene BNIP3 in ventricular myocytes. Circulation. 112:3777-3785.

63. Brocheriou, V., et al. 2000. Cardiac functional improvement by a human $\mathrm{Bcl}-2$ transgene in a mouse model of ischemia/reperfusion injury. J. Gene Med. 2:326-333.

64. Chen, Z., Chua, C.C., Ho, Y.S., Hamdy, R.C., and Chua, B.H. 2001. Overexpression of Bcl-2 attenuates apoptosis and protects against myocardial I/R injury in transgenic mice. Am. J. Physiol. Heart Circ. Physiol. 280:H2313-H2320.

65. Cohn, J.N., Ferrari, R., and Sharpe, N. 2000. Cardiac remodeling - concepts and clinical implications: a consensus paper from an international forum on cardiac remodeling. Behalf of an International Forum on Cardiac Remodeling. J. Am. Coll. Cardiol.
35:569-582.

66. Yu, C.M., et al. 2005. Left ventricular reverse remodeling but not clinical improvement predicts long-term survival after cardiac resynchronization therapy. Circulation. 112:1580-1586.

67. Muller-Ehmsen, J., et al. 2002. Rebuilding a damaged heart: long-term survival of transplanted neonatal rat cardiomyocytes after myocardial infarction and effect on cardiac function. Circulation. 105:1720-1726.

68. Anversa, P., et al. 2007. Concise review: stem cells, myocardial regeneration, and methodological artifacts. Stem Cells. 25:589-601.

69. Syed, F., Diwan, A., and Hahn, H.S. 2005. Murine echocardiography: a practical approach for phenotyping genetically manipulated and surgically modeled mice. J. Am. Soc. Echocardiogr. 18:982-990.

70. Syed, F., et al. 2004. Physiological growth synergizes with pathological genes in experimental cardiomyopathy. Circ. Res. 95:1200-1206.

71. Guo, Y., et al. 1998. Demonstration of an early and a late phase of ischemic preconditioning in mice. Am. J. Physiol. 275:H1375-H1387.

72. Ren, X., Wang, Y., and Jones, W.K. 2004. TNF-alpha is required for late ischemic preconditioning but not for remote preconditioning of trauma. J. Surg. Res. 121:120-129.

73. Yang, Z., Berr, S.S., Gilson, W.D., Toufektsian, M.C., and French, B.A. 2004. Simultaneous evaluation of infarct size and cardiac function in intact mice by contrast-enhanced cardiac magnetic resonance imaging reveals contractile dysfunction in noninfarcted regions early after myocardial infarction. Circulation. 109:1161-1167.

74. Weiss, C.R., et al. 2003. Stunned, infarcted, and normal myocardium in dogs: simultaneous differentiation by using gadolinium-enhanced cine MR imaging with magnetization transfer contrast. Radiology. 226:723-730.

75. Lee, S.S., et al. 2003. MR imaging of reperfused myocardial infarction: comparison of necrosisspecific and intravascular contrast agents in a cat model. Radiology. 226:739-747.

76. Barkhausen, J., Ebert, W., Debatin, J.F., and Weinmann, H.J. 2002. Imaging of myocardial infarction: comparison of magnevist and gadophrin-3 in rabbits. J. Am. Coll. Cardiol. 39:1392-1398.

77. Milei, J., and Storino, R. 1986. Early myocardial infarction. A feasible histologic diagnostic procedure. Jpn. Heart J. 27:307-319.

78. Sanbe, A., et al. 2003. Reengineering inducible cardiac-specific transgenesis with an attenuated myosin heavy chain promoter. Circ. Res. 92:609-616. 